\title{
Inmunoterapia en astrocitomas de alto grado: principios y estado actual
}

\author{
D. Pinilla-Arias; O. Mateo-Sierra; F.A. Gutiérrez; C. Fernández-Carballal y R. Carrillo
}

Servicio de Neurocirugía. Hospital General Universitario Gregorio Marañón. Madrid.

\section{Resumen}

Desde hace tiempo se conoce que la presencia de un tumor origina en el organismo una reacción del sistema inmune enfocada a eliminar la amenaza que el desarrollo tumoral implica. Sin embargo, se van averiguando diferentes mecanismos por los cuales los tumores provocan un estado de inmunosupresión que dificulta el desarrollo de una reacción inmunitaria eficaz. La utilización del sistema inmune como arma frente a los astrocitomas de alto grado constituye una de las principales estrategias de investigación desde los primeros ensayos de Bloom en 1960, siendo hoy en día una práctica habitual en la terapia antineoplásica de múltiples disciplinas médicas.

El objetivo de este estudio es describir las características de las reacciones inmunológicas del sistema nervioso central, los mecanismos utilizados por los tumores astrocitarios para despistar las defensas del organismo y las diferentes líneas de investigación llevadas a cabo hasta la fecha, mostrando sus bases fisiopatológicas y los resultados obtenidos, basándonos en una extensa revisión bibliográfica.

PALABRAS CLAVE: Inmunoterapia. Tratamiento de gliomas. Citoquina. Linfocitos.

Immunotherapy in high grade astrocytomas: principles and current state

\section{Summary}

It is generally accepted that tumour development promotes a systemic response leading to protect the host against cancer. However, tumours may as well elicit a partial immunodeficiency to avoid the development of a complete and active immune response. Since Bloom's first studies on immunotherapy to treat high grade gliomas in 1960, many attempts have been made from

Recibido: 29-09-04. Aceptado: 19-11-04 different medical specialties to use the immune system as a weapon against a great diversity of cancers.

Main objective of this study is to outline the basic features of the immune response inside the Central Nervous System, the strategies employed by astrocytic tumours to evade body defences, and to provide an extended literature review on research on immunotherapy, especially concerning its patho-physiology and the clinical results achieved till date.

KEY WORDS: Immunotherapy. Glioma treatment. Interleukin Lymphocytes

\section{Introducción}

Desde que en 1883 el Dr. Rickman Godlee realizara la primera intervención quirúrgica sobre un astrocitoma ${ }^{19}$, se han llevado a cabo múltiples intentos para mejorar la supervivencia y la calidad de vida de los pacientes con este tipo de tumores. Las pautas tradicionales de tratamiento han conseguido una eficacia muy limitada y los resultados publicados en la literatura son decepcionantes. Por ello, se han desarrollado numerosas estrategias terapéuticas desde la biología molecular, la genética, la inmunología y otras disciplinas científicas. Así, en 1960, Bloom y colaboradores efectuaron el primer intento de vacunación contra un glioblastoma multiforme al realizar el implante subcutáneo de células tumorales ${ }^{7,8}$. De un modo complementario,

Abreviaturas. ADCC: respuesta celular mediada por anticuerpos. APC: celúlas presentadoras de antígeno. ASL: linfocitos autólogos estimulados. BCG: bacilo de Calmette-Guérin. BHE: barrera hematoencefálica. CMH: complejo mayor de histocompatibilidad. EFGr: receptor de factor de crecimiento epidérmico. GM-CSF: factor de crecimiento de colonias de granulocitos y monocitos. HLA: antígeno leucocitario humano. IFN: interferón. IGF: factor de crecimiento similar a la insulina. IL: interleukina. LAK: células citotóxicas activadas por linfoquinas. MAK: células citotóxicas activadas por mitógenos. NK: natural killer. TGF: factor de crecimiento transformante. Th: linfocitos Thelper. TIL: lifocitos infiltrantes de tumor. TNF: factor de necrosis tumoral. 
Scheinberg y Taylor, en 1968, llamaron la atención sobre la importancia de la terapia inmunológica en el tratamiento de los tumores de estirpe astrocitaria ${ }^{93}$. A partir de entonces se ha avanzado mucho en el conocimiento del comportamiento biológico e inmunológico de dichas neoplasias y se han abierto numerosos frentes de trabajo que están consiguiendo éxitos aislados ${ }^{5,6,108}$. En esta revisión pretendemos describir las diferentes tendencias de trabajo en inmunoterapia y los resultados obtenidos con ellas así como anticipar algunas de las nuevas estrategias sobre las que se está trabajando.

\section{El sistema nervioso central: ¿un lugar privilegiado?}

El SNC tiene una manera única de reaccionar frente a las agresiones microbiológicas y tumorales que ha llevado a considerarlo como un lugar privilegiado desde un punto de vista inmune, ya que el desarrollo de una reacción inmunológica completa podría tener unas consecuencias demasiado graves para el cerebro ${ }^{64}$. El parénquima cerebral no impide el desarrollo de reacciones inmunes pero sí precisa un mayor estímulo. Así, por ejemplo, en sus estudios con la inoculación de BCG, Matyszak y Perry ${ }^{63}$, demostraron que la respuesta en el sistema nervioso central requería un mayor volumen de inóculo y nunca fue tan efectiva, comparada con la respuesta en otros órganos de la economía. Otros indicios de este privilegio se vieron reflejados en los experimentos de Medawar con implantes celulares, en los que dicho autor demostró la larga supervivencia de los implantes en el $\mathrm{SNC}^{65}$.

El primer obstáculo que se encuentra la respuesta inmune es la barrera hematoencefálica (BHE), con propiedades específicas que dificultan la entrada de los diferentes componentes del sistema inmune. Por un lado, las uniones íntimas u occludens (tight junctions) entre las células endoteliales limitan en extremo la difusión paracelular ${ }^{10}$. De manera adicional, las células endoteliales tienen un flujo intracelular limitado por la carencia de vesículas de pinocitosis, a la vez que precisan de sistemas específicos de transporte para la incorporación de metabolitos y la eliminación de residuos. La consecuencia de todo esto es que la concentración de inmunoglobulinas y complemento es mucho menor que las encontradas en el plasma y que el tráfico de células inflamatorias está más limitado que en otros órganos ${ }^{35}$.

Un segundo inconveniente es la ausencia de un sistema convencional de drenaje linfático en el $\mathrm{SNC}^{64,113}$, lo que limita el contacto de los antígenos con el sistema linfático cervical ${ }^{11} \mathrm{y}$ dificulta el desencadenamiento de una reacción inmune completa. Sin embargo, dicha ausencia de drenaje no es absoluta ya que se ha demostrado la llegada de proteínas marcadas a los ganglios linfáticos cervicales desde el cerebro ${ }^{114}$.

Otro obstáculo al desarrollo de respuestas inmunes competentes en el SNC es la escasa expresión de moléculas del $\mathrm{CMH}$, tanto en células gliales, como en neuronas y en células de microglía en condiciones de reposo ${ }^{31}$, aunque la expresión puede aumentar de manera significativa en caso de ser estimuladas con citokinas, especialmente en el caso de influencia tumoral ${ }^{101}$.

Por último, en el parénquima cerebral, la interacción entre las células del sistema inmune y las llamadas células presentadoras de antígeno es mucho menos eficaz que en el resto del organismo debido a que, en el sistema nervioso la microglia, que es el subgrupo celular que representa dicho papel, necesita activarse para cumplir correctamente su función, retrasando y, en ocasiones, imposibilitando la reacción adecuada. A esto se le añade el hecho de que las respuestas que se producen en el sistema nervioso central son con frecuencia del tipo Th2 (humoral), con lo que disminuye la eficacia inmunológica, o bien son incompletas por la falta de moléculas adyuvantes desencadenando anergia o tolerancia al antígeno.

En definitiva, el sistema nervioso mantiene un lugar inmunoprivilegiado, estando sometido a un riguroso control de la inflamación. Las respuestas inmunes se producen y desencadenan como en el resto del organismo, aunque con mayor limitación.

\section{Inmunología de los astrocitomas}

Los tumores astrocitarios son tumores casi siempre confinados al SNC, con lo que representan un desafío a la inmunovigilancia sistémica por el hecho de estar "protegidos" por la BHE y los mecanismos de regulación de la reacción inmune. Sin embargo, es un hecho común encontrar infiltrados linfocitarios alrededor de los tumores astrocitarios, aunque el valor pronóstico de este hallazgo es controvertido $^{12,76}$.

En general, los pacientes afectados de tumores astrocitarios muestran importantes alteraciones en la función inmune, habiéndose encontrado un cierto grado de anergia cutánea e hipersensibilidad retardada alterada ${ }^{11,60,61}$, una disminución de los linfocitos $T$ circulantes ${ }^{61}$, una reducción de la respuesta de los linfocitos a mitógenos ${ }^{9,11}$, una disminución de la respuesta de anticuerpos ${ }^{61} \mathrm{y}$ una citotoxicidad mediada por células $\mathrm{T}$ deficiente ${ }^{11,61}$. El hecho de que la citorreducción tumoral mejore de manera significativa la función inmune ${ }^{13}$ apoya la teoría de que la presencia del tumor es en sí misma el principal factor causal del estado de inmunosupresión ${ }^{60}$.

Se han encontrado diferentes mecanismos por los cuales los astrocitomas consiguen evadir la vigilancia del sistema inmune. Estos mecanismos son de tipo humoral y de tipo celular y se resumen en la tabla 2 y en la figura 1.

\subsection{Mecanismos humorales de escape}




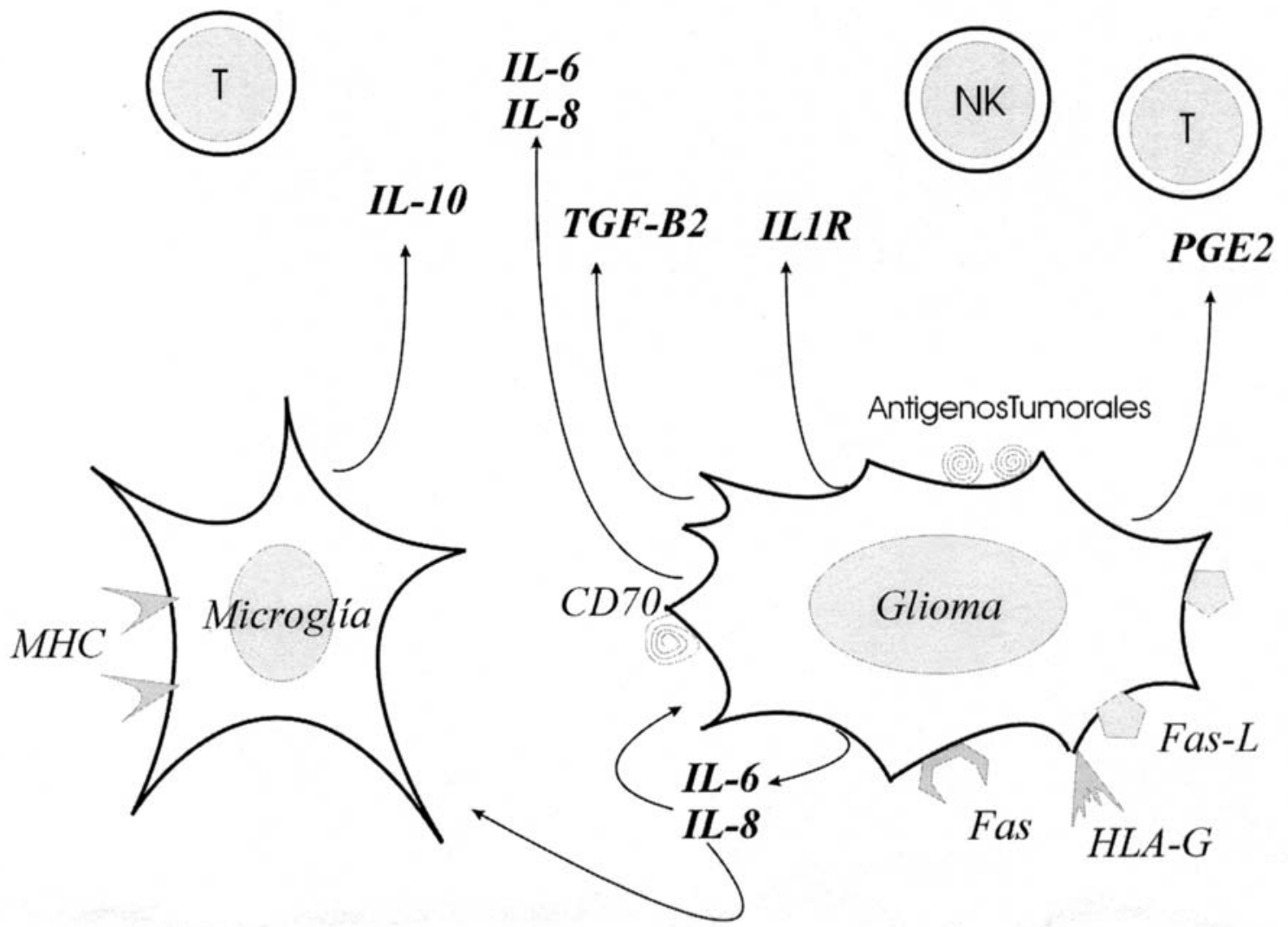

Figura 1: Mecanismos de inmunosupresión tumoral. CMH: Complejo Mayor de Histocompatibilidad; PGE2: Prostaglandina E2; IL: Interleuquina; TGF: Factor de Crecimiento Transformante; NK: Natural Killers.

Tabla 1

Fisiología de la respuesta inmune en el SNC

\begin{tabular}{|l|}
\hline \multicolumn{1}{|c|}{ INMUNOPRIVILEGIO Sistema Nervioso } \\
\hline - Barrera Hematoencefálica. \\
- Escasez de drenaje linfático. \\
- Escasa expresión de moléculas del CMH. \\
- Respuesta poco eficaz de las células presentadoras de antígeno. \\
\hline
\end{tabular}

$\mathrm{CMH}$ (Complejo Mayor de Histocompatibilidad)

Desde hace tiempo se conoce la existencia del TGF$\beta 2$ (factor de crecimiento transformante, antes llamado factor de supresión de células T derivado de glioblastoma). Esta sustancia inhibe la proliferación de los linfocitos T y $\mathrm{B}^{38,47}$, la expresión de receptores de IL- $2^{48}$, la producción de citoquinas estimuladoras de la respuesta inmune ${ }^{80}$ y la activación de las células Natural Killer (NK) y Presentadoras de Antígeno (APC) $)^{39}$. Además, disminuye la expresión de los antígenos MHC clase II HLA-DR, contribuyendo a hacer menos eficaz la respuesta antitumoral ${ }^{115}$. De modo adicional, el TGF- $\beta 2$ está implicado en la angiogénesis y en la estimulación del crecimiento tumoral ${ }^{43}$. Apoyando estos datos, existen experimentos con un inhibidor de este factor, la decorina, que han demostrado una supresión del crecimiento tumoral con efecto inmunoestimulador en diferentes estudios in vivo e in vitro ${ }^{72,95}$.

Otros factores humorales implicados son las prostaglandinas, especialmente la PGE2, que actúa como potente 
Tabla2

Mecanismos tumorales de escape

\begin{tabular}{|ll|}
\hline Humorales & Celulares \\
\hline TGF- $\beta$ & Disminución de expresión de MCH I \\
PGE2 & Fas/Fas-L \\
IL10 & Expresión de HLA-G \\
IL6 e IL8 & Expresión de CD70 \\
IL1-R & Inhibición de células NK \\
\hline
\end{tabular}

TGF- $\beta$ (Factor de Crecimiento Transformante Beta); PGE2 (Prostaglandina E2); IL (Interleukina); IL1-R (Receptor de Interleukina 1); MHC (Complejo Mayor de Histocompatibilidad); HLA (Antígeno Leucocitario Humano); NK (Natural Killers).

inhibidor de la proliferación linfocitaria ${ }^{54}$, y diferentes tipos de interleuquinas, entre las que nos encontramos: 1) La IL10 que inhibe la liberación de Interferón gamma (IFN$\gamma)$ por los linfocitos, del Factor de Necrosis Tumoral alfa (TNF- $\alpha$ ) por los monocitos y la expresión de $\mathrm{CMH}$ de clase II ${ }^{88}$; 2) La IL6 y la IL8 que actúan como promotores del crecimiento tumoral al estimular la liberación de factores de crecimiento ${ }^{75}$ y desvían la respuesta hacia una reacción humoral; 3) El receptor soluble de IL1 que distorsiona el desencadenamiento de la respuesta fisiológica.

\subsection{Mecanismos celulares de escape}

El mecanismo más simple, por el cual la célula tumoral elude la inmunovigilancia celular, es la escasa expresión de moléculas $\mathrm{CMH}$ clase I, por lo que es difícil reconocerlas como células ajenas al organismo.

Uno de los mecanismos de escape celular mejor estudiados es la expresión de Fas (CD95), una proteína de la familia del factor de necrosis tumoral y su ligando (FasL) en las células tumorales. Esta proteína induce apoptosis de linfocitos T tanto CD4 como CD8 ${ }^{103}$ y su efecto está potenciado en aquellos medios en los que encuentre TGF- $\beta^{16}$.
Además, algunos tumores astrocitarios expresan HLA-G, un subtipo atípico de CMH de clase I que se encuentra en algunos tejidos normales como la placenta, pero también en algunos tumores y parece actuar inhibiendo la función de las células NK y los linfocitos $\mathrm{T}^{104}$. Finalmente, se han encontrado múltiples astrocitomas que expresan en sus membranas CD70, proteína transmembrana cuya activación promueve la apoptosis de los linfocitos tanto T como $\mathrm{B}^{33}$.

Por otro lado, las células tumorales producen una inhibición de la función de las células NK igualmente por mecanismos humorales y celulares, haciendo menos eficaz la respuesta natural antitumoral ${ }^{25}$.

En resumen, los enfermos con astrocitomas están sumidos en un estado de inmunosupresión, tanto local como sistémica, que favorece una escasa y poco eficaz respuesta inmune frente al crecimiento tumoral. Basándose en estos datos, la potenciación de la función inmune podría resultar efectiva como tratamiento antitumoral.

\section{Terapias inmunológicas}

El campo de la inmunología ha desarrollado terapias

\section{Tabla 3}

Estrategias de Inmunoterapia

\begin{tabular}{|c|c|}
\hline Terapias NO específicas & \multirow{6}{*}{$\begin{array}{l}\text { Otras estrategias experimentales } \\
\text { - Vacunas por terapia inmunogénica. } \\
\text { - Terapia inmunogénica local } \\
\text { - Función de células NK } \\
\text { - Reversión de Inmunosupresión inducida } \\
\text { por el tumor }\end{array}$} \\
\hline - Inmunoestimulantes & \\
\hline - Terapia con citoquinas & \\
\hline Inmunoterapia pasiva específica & \\
\hline Inmunoterapia adoptiva & \\
\hline Inmunoterapia activa & \\
\hline
\end{tabular}

NK (Natural killers) 
muy variadas con el fin de utilizar al sistema inmune como herramienta en el tratamiento de los tumores. Resumimos una clasificación de las diferentes terapias en la tabla 3. Diferentes disciplinas clínicas cuentan con terapias inmunes en sus protocolos de actuación, a pesar de que sigue siendo necesario continuar con las diferentes líneas de investigación.

Los principales problemas con los que se ha encontrado tradicionalmente la inmunoterapia son el riesgo de desencadenamiento de una reacción inflamatoria masiva y por consiguiente letal, la ausencia de antígenos verdaderamente específicos de tumor, la limitación en el conocimiento de la interacción entre el sistema inmune y los gliomas, y la ausencia de un modelo animal verdaderamente representativo del que se puedan extraer consecuencias significativas.

\subsection{Terapias no especificas}

\subsubsection{Sustancias Inmunoestimulantes}

Este tipo de terapias ha sido experimentado y empleado ampliamente en el tratamiento de otros tumores, principalmente el melanoma y el carcinoma de vejiga urinaria. Se han empleado como estimulantes más importantes el BCG (bacilo de Calmette-Guérin) y el Corynebacterium parvum (OK432). Se postula como mecanismo de acción inmunoestimulante la capacidad de estos microorganismos de favorecer el desencadenamiento de una activación policlonal de células inmunes principalmente estimulando a las células $\mathrm{NK}^{71}$. Estas estrategias han tenido una escasa repercusión clínica como terapias aisladas en los tumores cerebrales, pero su uso se podría revitalizar por su posible utilidad como terapia de potenciación de la respuesta a vacunas antitumorales.

\subsubsection{Terapia con citoquinas}

En ensayos preclínicos ${ }^{41,55,75}$ se comprobó un aumento de la función y eficacia de los linfocitos $\mathrm{T}$ citotóxicos contra tumores cerebrales al estimular a los pacientes con IL2, IL7, IL12, IL18, ,... Por ello, las citoquinas comenzaron a utilizarse por vía intravenosa aunque su uso se vio muy limitado por la gran cantidad de efectos secundarios sistémicos que producía ${ }^{45,68}$. Para evitar esta complicación, se han desarrollado diferentes vías de administración ${ }^{50,91}$. En los últimos años se ha propuesto como alternativa la utilización de células programadas genéticamente, para producir la liberación prolongada y mantenida de las sustancias deseadas mediante la transducción de genes de citoquinas $^{58,107}$.

La IL2 es una molécula fundamental en la activación y posterior acción de los linfocitos $\mathrm{T}$. Se ha empleado en múltiples ensayos clínicos con el principio de aumentar la actividad de los linfocitos $\mathrm{T}$ citotóxicos y de promover la función de las células NK, pero su actividad terapéutica requiere altas dosis, lo cual limita su uso por vía sistémica debido a los efectos secundarios ${ }^{68,87}$. Se han desarrollado otras vías de administración y se utiliza en múltiples protocolos, tanto como terapia única como en combinación con quimioterapia $^{91}$, o terapias celulares ${ }^{50}$. Más recientemente, se ha publicado el desarrollo de biopolímeros de liberación controlada de esta citoquina ${ }^{83}$, y el desarrollo de células modificadas genéticamente para producirla de manera continuada y prolongada ${ }^{29}$. Los resultados de los diferentes estudios han sido muy controvertidos, no habiéndose encontrado una mejoría significativa de la supervivencia de los pacientes salvo éxitos aislados ${ }^{22}$.

La IL4 es otra de las citoquinas sobre las que se ha investigado intensamente. Se han encontrado resultados prometedores en los ensayos de distribución local mediante células modificadas ${ }^{3,4,109}$, pero se han comunicado indicios de gran toxicidad local por inducción de edema cerebra ${ }^{98}$.

Otras moléculas que han demostrado eficacia en los estudios experimentales son: la IL12, utilizada con éxito tanto en administración sistémica ${ }^{14,107}$ como en depósito intracavitario ${ }^{96}$, mostrando potenciación de la inmunidad celular y promoción de la formación de IFN- $\gamma$; el factor de crecimiento de colonias de granulocitos y monocitos (GM-CSF) molécula iniciadora de la respuesta inmune que estimula la liberación de IL4 e IFN- $\gamma$ y con la que se han obtenido resultados prometedores al combinarla con vacunas modificadas genéticamente ${ }^{34}$.

Las terapias con interferones se han investigado durante muchos años con resultados muy controvertidos. A pesar de haberse mostrado como potentes agentes antitumorales in vitro los resultados en los ensayos clínicos no han conseguido reproducir la misma eficacia.

El IFN- $\alpha$ fue empleado por Jereb y colaboradores ${ }^{44}$ en 1989 con resultados positivos en 17 pacientes, al igual que en el estudio de Merchant en 1992 en combinación con IL2 ${ }^{69}$, pero los estudios de Vaquero y colaboradores únicamente con IFN- $\alpha$ o en combinación con terapia celular no consiguieron reproducir dichos éxitos terapéuticos ${ }^{62,102}$. Sin embargo, evidencias recientes parecen reflejar sinergismo en su acción junto a radioterapia o quimioterapia intracavitaria o sistémica ${ }^{15,21}$.

El IFN- $\beta$ ha demostrado gran actividad antitumoral in vitro $^{111} \mathrm{e}$, incluso, se ha demostrado buena respuesta en estudios clínicos ${ }^{111,112}$, pero aún debe establecerse la dosis adecuada y la verdadera utilidad clínica.

El IFN- $\gamma$ es el que más expectativa creó inicialmente por el hecho de que parte de la inmunosupresión provocada por el tumor se debe a la interferencia en los mecanismos de acción de esta molécula. Así, en 1994, Fakkila y colaboradores llevaron a cabo un ensayo clínico aleatorizado y 
Tabla 4

Antígenos tumorales

\begin{tabular}{|l|l|l|l|}
\hline $\begin{array}{l}\text { Antígenos } \\
\text { Silentes }\end{array}$ & $\begin{array}{l}\text { Atígenos } \\
\text { Tejido-específicos }\end{array}$ & Virus & Oncogenes \\
\hline MAGE & Tirosinasa & E6, E7 & EFG-R mutado \\
BAGE & Gp-100 & & Bcr-abl \\
GAGE & PSA & P53, ras \\
Tenascina & & & \\
\hline
\end{tabular}

PSA (Antígeno Prostático Específico); EFG-R (Receptor de Factor de Crecimiento Epidérmico).

randomizado en catorce pacientes, comparándolos con un grupo control y se demostró la seguridad de la sustancia en administración intracavitaria pero no se consiguieron objetivar diferencias en la supervivencia de los pacientes ${ }^{26}$.

\subsection{Inmunoterapia pasiva especifica}

Con este término se hace referencia al empleo de anticuerpos dirigidos contra las células tumorales. La ventaja de este tipo de terapias radica en la capacidad de estas moléculas de dirigir la respuesta inmune contra las células tumorales, pudiendo añadir, además, agentes citolíticos contra las células tumorales y disminuir la inmunosupresión tumoral dependiente de factores solubles. El principal inconveniente radica en el hecho de no disponer de anticuerpos realmente específicos contra las neoplasias y en la dificultad de hacer llegar suficiente cantidad de los mismos al lecho tumoral, aparte de la capacidad de dichas moléculas de provocar reacciones adversas contra el huésped.

Los anticuerpos sobre los que se han centrado las investigaciones han sido principalmente las dirigidas contra moléculas que se expresan con más frecuencia en las células tumorales, aquellas que en condiciones normales no se expresan en el tejido huésped, los que son antígenos específicos de tejidos concretos o que son el resultado de mutaciones en oncogenes ${ }^{20}$ (ver tabla 4).

En su estudio con anticuerpos marcados con yodo radiactivo, Miyamoto y colaboradores emplearon como diana el receptor mutado de EGF, que es expresado en un porcentaje significativo de glioblastomas, especialmente de tipo primario. Demostraron la seguridad de la administración sistémica del mismo y la respuesta favorable de algunos de los pacientes tratados ${ }^{70}$.

Los estudios más recientes ${ }^{5,6,84,86}$ se inclinan por la administración local de los anticuerpos como vía de administración empleando como antígeno diana la tenascina (un antígeno relativamente específico de tejido astrocitario). En su estudio, Bigner y colaboradores demostraron una tasa de respuesta inicial del $51 \%$ y tres de cinco astrocitomas anaplásicos permanecieron sin resto tumoral a los treinta meses de seguimiento ${ }^{5,6}$. En estos estudios también se habla como posible diana la gp240, habiéndose obtenido resultados similares ${ }^{5,6}$. En la tabla 5 mostramos los resultados de los ensayos clínicos publicados y los resultados obtenidos.

3.2.1. Nuevas estrategias en terapia con anticuerpos monoclonales

Los resultados prometedores con los ensayos preclínicos y la capacidad de los anticuerpos de vehicular sustancias citotóxicas ha estimulado la realización de múltiples ensayos experimentales empleando la tecnología de hibridación con el objetivo de optimizar el efecto lítico de los anticuerpos.

Una de las técnicas utilizadas consiste en la conjugación de inmunoglobulinas con venenos conocidos como es el caso de la toxina de la Pseudomonas aeruginosa ${ }^{79} \mathrm{o}$ bien la toxina del Corynebacterium difteriae ${ }^{56}$, con resultados muy alentadores tanto en lo referente a la respuesta inicial del tumor como a la supervivencia posterior. Otra estrategia emergente es la basada en el concepto de anticuerpos biespecíficos, que son aquellos que tienen la cualidad de poder anclarse por un lado a antígenos tumorales específicos y, por otro, a antígenos linfocitarios activadores (fig. 2), de manera que la respuesta inmune pueda dirigirse de un modo más eficaz contra la diana elegida. Principalmente se han usado fracciones anti-CD3 como elemento de anclaje a linfocitos $y$ fracciones anti-EFGrIII como elemento antitumoral, habiéndose obtenido buenos resultados preliminares en estudios aislados ${ }^{46,74}$.

\subsection{Inmunoterapia adoptiva}

Esta estrategia consiste en el empleo de linfocitos periféricos activados en cultivo con IL2, por lo que se les denomina células LAK (Lymphokine Activated Killer cells). Son células inespecíficas y lo que se consigue en el labora- 
Neurocirugía

Inmunoterapia en astrocitomas de alto grado: principios y estado actual

2005; 16:345-358

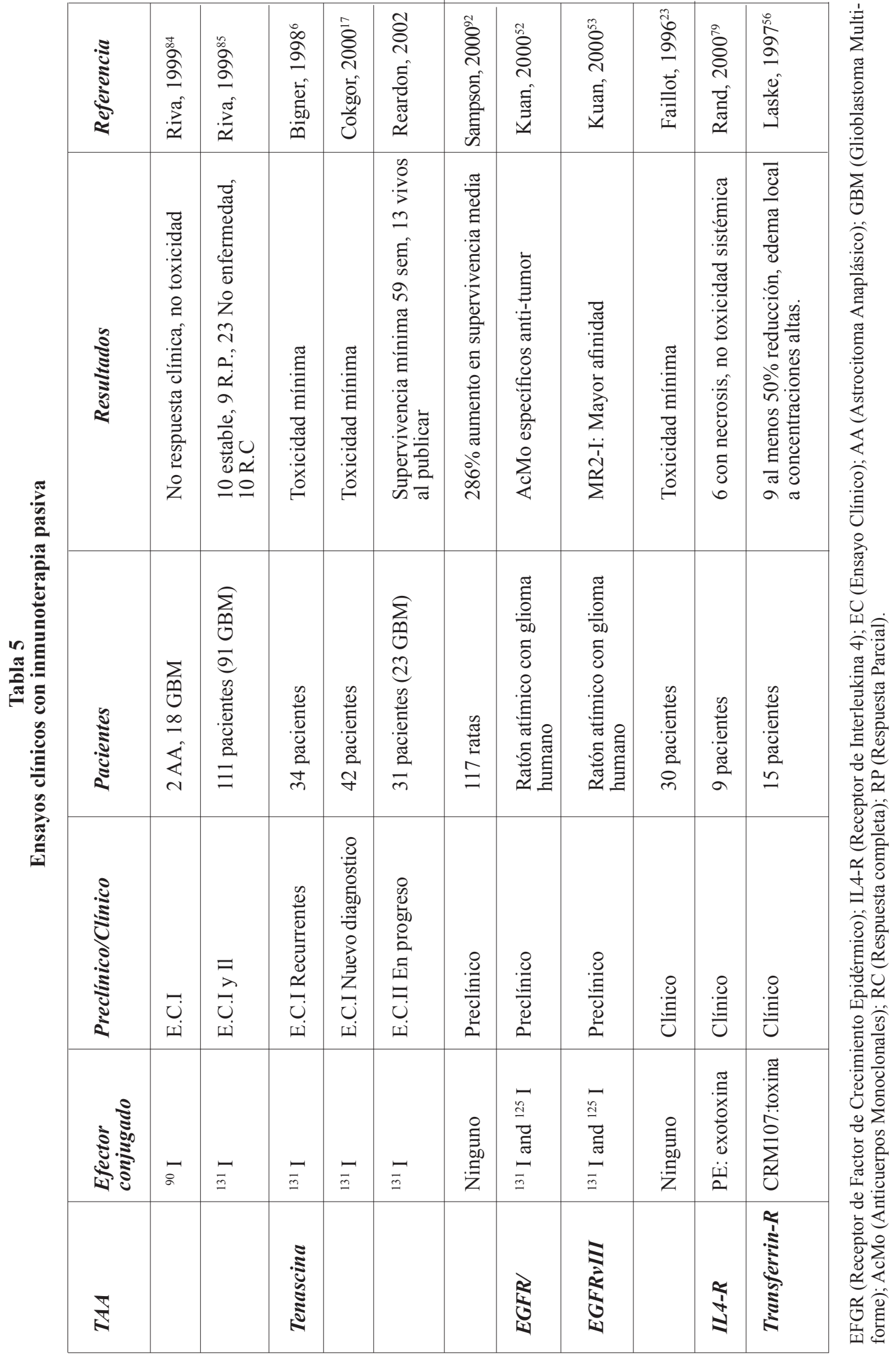




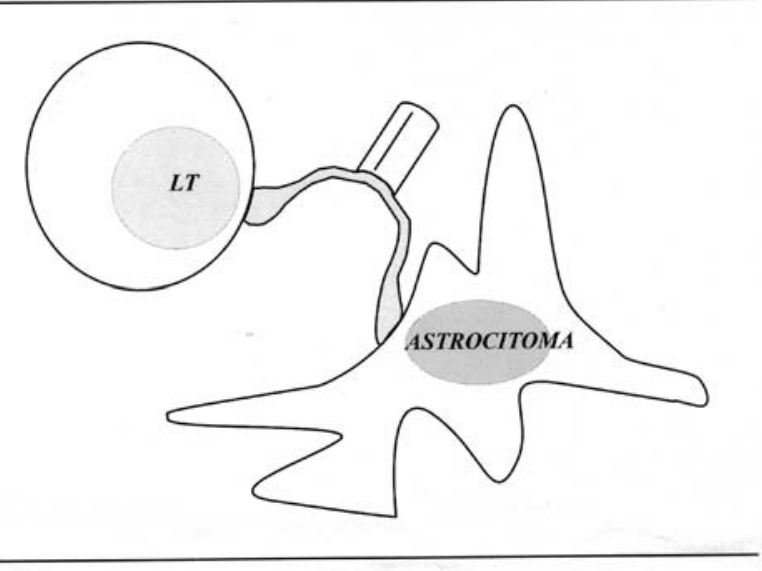

Figura 2: Anticuerpos biespecificos. LT: Linfocitos $T$.

torio es una expansión no clonal de dichos linfocitos. Tras la expansión en el laboratorio de los linfocitos de sangre periférica, estos se depositan en la cavidad de resección tumoral. Se han realizado múltiples estudios tanto in vitro como in $v i v o^{2,32,50,66,67}$, no habiéndose obtenido resultados significativos salvo en casos aislados ${ }^{32}$. Este escaso éxito de la inmunoterapia adoptiva tradicional puede explicarse por múltiples motivos, como son el estado de inmunosupresión de los linfocitos del paciente, los tratamientos corticoideos asociados y su efecto linfosupresor, o la habilidad tumoral para difuminar la acción antitumoral de los linfocitos acti$\operatorname{vados}^{50}$. Con objeto de mejorar la respuesta linfocitaria y, con ello, aumentar la eficacia tumoricida de los linfocitos, se han investigado diferentes estrategias experimentales.

Por un lado, se aprovechó la capacidad de ciertas lectinas como la fitohemaglutinina o la concanavalina A de estimular la proliferación y activación de los linfocitos $\mathrm{T}$ para tratar de generar las denominadas células MAK (Mitogenic Activated Killer Cells) o ASL (Autologous Stimulated Lymphocytes) ${ }^{40}$. A pesar de unos resultados esperanzadores en el laboratorio, la eficacia en ensayos clínicos no ha respondido a las expectativas y la supervivencia obtenida ha sido similar a los controles históricos ${ }^{42}$.

Las células TIL (Tumor-Infiltrating Lymphocytes) han sido otra de las dianas de las investigaciones, con la premisa de que los linfocitos encontrados en la pieza tumoral podrían producir una respuesta inmune mucho más específica y efectiva contra el tumor, lo cual se demostró con éxito en estudios in vitro ${ }^{86}$. Una vez más, los resultados clínicos han sido decepcionantes ${ }^{113}$ ya que se ha demostrado que dichos linfocitos tienen disminuido su potencial proliferativo así como una menor capacidad citolítica que los linfocitos periféricos. Además, el aislamiento de dichos linfocitos es técnicamente difícil por representar menos del $1 \%$ de la muestra tumoral.

La inmunoterapia adoptiva específica ha supuesto un nuevo intento de mejora de la función de los linfocitos. Consiste en la inmunización previa del paciente con extracto tumoral (asociado a estimuladores inespecíficos de la inmunidad como BCG o GMCSF), cultivo de linfocitos $\mathrm{T}$ activados tras la vacuna y posterior reinfusión de dichos linfocitos. Los resultados obtenidos con estas técnicas tampoco han supuesto un aumento de la supervivencia de los pacientes ${ }^{36,77}$.

Kruse y colaboradores ${ }^{50}$ han estudiado la posibilidad de emplear linfocitos reactivos frente a proteínas del $\mathrm{CMH}$ basándose en la teoría de que estas moléculas se expresan con menor intensidad en neuronas y glía que en células tumorales, habiendo obtenido resultados clínicos prometedores. En un reciente estudio de los mismos autores se evalúa la eficacia de emplear con este fin células leucémicas no restringidas por el CMH (células TALL-104) con muy buenos resultados en animales de laboratorio ${ }^{51}$.

\subsection{Inmunoterapia activa: vacunación}

La utilización de vacunas antitumorales es una modalidad terapéutica muy extendida en el estudio de otras extirpes neoplásicas como es el caso del melanoma ${ }^{71}$. La existencia una reacción inmune frente a las células de astrocitoma y los resultados esperanzadores de diversas terapias inmunes han estimulado el desarrollo de líneas de investigación para potenciar la reacción inmune antitumoral.

La vacuna de Bloom $^{7,8} \mathrm{o}$ los experimentos de Trouillas ${ }^{100}$ fueron las primeras terapias de este tipo empleadas, obteniendo resultados dispares. Los principales inconvenientes de este tipo de terapias son la escasa respuesta provocada por la inmunosupresión inducida por el tumor y el riesgo potencial de encefalitis grave ante una respuesta frente a elementos neuronales o gliales. Es por ello que se han desarrollado dos nuevos frentes de experimentación con el fin de potenciar la respuesta y dirigirla de una manera más eficiente:

\subsubsection{Vacunación con células dendríticas}

Existen numerosos estudios ${ }^{57,107,108,110}$ que han demostrado que la inmunoterapia sistémica mediante el uso de células dendríticas es capaz de inducir una respuesta antitumoral en el tejido cerebral. En estos estudios se extraen células dendríticas y se cultivan junto a muestras tumorales inactivadas, para posteriormente, reintroducirlas en los pacientes. Yu y colaboradores consiguieron demostrar en 9 pacientes un aumento significativo de la supervivencia con mínima toxicidad ${ }^{110}$.

Basándose en los buenos resultados obtenidos en ensayos clínicos de neoplasias extracraneales ${ }^{37,73,97}$, Yamanaka y colaboradores $^{108}$ llevaron cabo un ensayo clínico I/II utilizando células dendríticas estimuladas con restos tumorales 
autólogos con posterior reintroducción por vía sistémica e intratumoral demostrando la seguridad del método y la capacidad de esta técnica para generar una respuesta inmune dentro del sistema nervioso.

\subsubsection{Terapia adoptiva inmunogénica}

Bajo este término se engloban las técnicas que se basan en la modificación genética de células con el fin de potenciar la respuesta inmune. En los diferentes ensayos publicados se han utilizado fibroblastos ${ }^{94}$, células dendríticas ${ }^{107}$ u otros vehículos como bacterias ${ }^{58}$ o células tumorales modificadas $^{82}$. Las modificaciones genéticas inducidas han supuesto la síntesis y liberación de citoquinas inmunoestimuladoras ${ }^{94,107,109} \mathrm{o}$ de factores que contrarrestan los efectos inmunosupresores del tumor ${ }^{24} \mathrm{y}$, en algún caso, la génesis de factores coadyuvantes del sistema inmune con objeto de potenciar su función (p. e. IGF1) ${ }^{82}$.

En otras ocasiones, lo que se ha intentado es generar células alogénicas que expresen antígenos tumorales como el EFGr-III ${ }^{1}$ o crear vacunas que consten de extractos tumorales y células modificadas para sintetizar citoquinas $^{28}$.

En general, los resultados en el laboratorio con modelos animales han reflejado resultados ilusionantes pero aún falta por ver la eficacia que dichas técnicas alcanzan en la práctica clínica.

\subsection{Nuevas terapias experimentales}

3.5.1. Terapias enfocadas a potenciar la función de las células NK

Las células NK (Natural Killers) forman parte de la inmunidad innata antitumoral y han despertado gran interés por su capacidad para producir una respuesta citotóxica antitumoral no dependiente de antígenos específicos. Múltiples estrategias experimentales en desarrollo se centran en potenciar su reacción frente a las células tumorales y revertir la inmunosupresión a la que se ven sometidas por dichas células. Se están llevando a cabo en modelos de tumores extracraneales con buenos resultados en los ensayos realizados ${ }^{25}$. Entre las estrategias que se están desarrollando podemos encontrar: el uso de IL2 u otras citoquinas asociadas a anticuerpos monoclonales para promover la respuesta celular mediada por anticuerpos (ADCC) ${ }^{27,05}$; la introducción de células NK alogénicas con epítopos KIR discordantes $^{90} \mathrm{o}$ el bloqueo in vivo de la interacción KIRMHC clase I para promover la respuesta citolítica mediada por células NK anulando los mecanismos que favorecen la tolerancia inmune ${ }^{49}$.

\subsubsection{Terapias enfocadas a revertir la inmunosupresión}

$2005 ; 16: 345-358$

inducida por el tumor

Hasta la fecha, las estrategias desarrolladas con objeto de potenciar la respuesta antitumoral del sistema inmune han obtenido resultados clínicos discretos y se especula que la inmunosupresión mediada por las células tumorales es responsable en gran medida del relativo fracaso de estas terapias. Por esta razón, numerosos investigadores han centrado sus esfuerzos en el bloqueo de los factores tumorales responsables de dicha inmunosupresión. Se ha demostrado que el bloqueo mediante células T específicas ${ }^{30}$, anticuerpos monoclonale $^{89}$ o mediante sustancias específicas como la decorina $^{72,95}$ puede potenciar la respuesta y erradicar los tumores en animales de experimentación. Además, se ha demostrado que la manipulación local del microambiente tumoral mediante la disminución de la concentración de TGF- $\beta$ potencia la función citolítica de los linfocitos peritumorales ${ }^{60}$.

\section{Conclusiones}

El escaso éxito de las terapias convencionales en el tratamiento de los gliomas de alto grado ha potenciado el desarrollo de estrategias alternativas que progresivamente se van incorporando a la práctica clínica cotidiana. La inmunoterapia ha cosechado resultados muy dispares en lo referente a las diferentes estrategias de trabajo. Hasta ahora, el análisis de los resultados obtenidos en los diferentes estudios indica que no se ha podido demostrar un aumento de la supervivencia con las diferentes terapias. Sin embargo, los buenos resultados obtenidos en los estudios preclínicos, junto a los éxitos aislados, pero prometedores ,obtenidos en los ensayos clínicos, deben impulsar a mantener la investigación. Las terapias combinadas de factores, los ensayos con mecanismos bloqueantes de TGB- $\beta 2$ y los avances en el estudio de la inmunofisiología e inmunopatología son los elementos fundamentales para el desarrollo futuro de la inmunoterapia.

\section{Bibliografía}

1. Ashley, D.M., Sampson, J.H., Archer, G.E., Batra, S.K., Bigner, D.D., Hale, L.P.: A genetically modified allogeneic cellular vaccine generates MHC class I-restricted cytotoxic responses against tumor-associated antigens and protects against CNS tumors in vivo. J Neuroimmunol. 1997; 78: 3446.

2. Barba, D., Saris, S.C., Holder, C., Rosenberg, S.A., Oldfield, E.H.: Intratumoral LAK cell and interleukin-2 therapy of human gliomas. J Neurosurg. 1989; 70: 175182.

3. Benedetti, S., Bruzzone, M.G., Pollo, B. et al: Eradication of rat malignant gliomas by retroviral in vitro 
delivery of the interleukin 4 gene. Cancer Res. 1999; 59: 645-652.

4. Benedetti, S., Pirola, B., Pollo, B. et al: Gene therapy of experimental brain tumors using neural progenitor cells. Nat. Med. 2000; 6: 447-450.

5. Bigner, D.D., Brown, M., Coleman, R.E. et al.: Phase I studies of treatment of malignant gliomas and neoplastic meningitis with ${ }^{131}$ I-radiolabeled monoclonal antibodies antitenascin 81C6 and anti-chondroitin proteoglycan sulfate Me1-14 F(ab')2: A preliminary report. J Neurooncol. 1995; 24: 109-122.

6. Bigner, D.D., Brown, M.T., Friedman, A.H. et al.: Iodine-131-labeled antitenascin monoclonal antibody 81C6 treatment of patients with recurrent malignant gliomas: Phase I trial results. J Clin Oncol. 1998; 16: 2202-2212.

7. Bloom, H.J., Peckham, M.J., Richardson, A.E., Alexander, P.A., Payne, P.M.: Glioblastoma multiforme: A controlled trial to assess the value of specific active immunotherapy in patients treated by radical surgery and radiotherapy. Br J Cancer 1973; 27: 253-267.

8. Bloom, W.H., Carstairs, K.C., Crompton, M.R., McKissock, W.: Autologous glioma transplantation. Lancet 1960; 2: 77-78.

9. Bodmer, S., Strommer, K., Frei, K. et al.: Immunosuppression and Transforming Growth Factor-beta in glioblastoma: preferential production of transforming growth factor-beta 2. J Immunol. 1989; 143: 3222-3229.

10. Brightman, M.W., Reese, T.S.: Junctions between intimately apposed cell membranes in the vertebrate brain. J Cell Biol. 1969; 40: 648-677.

11. Brooks, W.H., Netsky, M.G., Normansell, D.E. et al.: Depressed cell-mediated immunity in patients with primary intracranial tumors: characterization of a humoral immunosuppressive factor. J Exp Med. 1972; 136: 1631-1647.

12. Brooks, W.H., Markesbery. W.R., Gupta, G.D., Roszman, T.L.: Relationship of lymphocyte invasion and survival of brain tumor patients. Ann Neurol. 1978; 4: 219-224.

13. Brooks, W.H., Latta, R.B., Mahaley, M.S. et al.: Immunobiology of primary intracranial tumors, part 5: correlation of a lymphocyte index and clinical status. J Neurosurg. 1981; 54: 331-337.

14. Brunda, M.J., Luistro, L., Warrier, R.R. et al: Antitumor and antimetastatic activity of interleukin 12 against murine tumors. J Exp Med. 1993; 178: 1223-1230.

15. Buckner, J.C., Brown, L.D., Kugler, J.W. et al.: Phase II evaluation of recombinant interferon alpha and BCNU in recurrent glioma. J Neurosurg. 1995; 82: 430-435.

16. Chen, J.J., Sun, Y., Nabel, G.J.: Regulation of the proinflammatory effects of Fas ligand (CD95L). Science 1998; 282: 1714-1717.

17. Cokgor, I., Akabani, G., Kuan, C.T. et al.: Phase I trial results of iodine-131-labeled antitenascin monoclonal antibody 81C6 treatment of patients with newly diagnosed malignant gliomas. J Clin Oncol. 2000; 18: 3862-3872.

18. Cserr, H. F., Knopf, P. M.: Cervical lymphatics, the blood-brain barrier and the immunoreactivity of the brain: a new view. Immunol. Today 1992; 13: 507-512.

19. Davis, C.H., Bradford, R.: A surgical history of Maida Vale Hospital. En: Walker, M.D., Thomas, D.G.T. (eds): Biology of brain tumor; Nijhoff, Boston, 1986.

20. Dietrich, P.Y., Walker, P.R., Calzascia, T., Tribolet, N.: Immunology of brain tumors and implications for immunotherapy. En Kaye, A.H., Laws, E.R.: Brain tumors $2^{\text {nd }}$ Ed., Churchill and Livingstone, 2003; Chapter 8: 135150 .

21. Dillman, R.O., Wiemann, M., Oldham, R.K. et al: Interferon $\alpha-2 \mathrm{a}$ and external beam radiotherapy in the initial management of patients with glioma: a pilot study of the national biotherapy study group. Cancer Biother. 1995; 10: 265-271.

22. Dunn, I.F., Black, P.M.: The neurosurgeon as local oncologist: cellular and molecular neurosurgery in malignant glioma therapy. Neurosurgery 2003; 52: 1411-1424.

23. Faillot, T., Magdelenat, H., Mady, E. et al: A Phase I study of an anti-epidermal growth factor receptor monoclonal antibody for the treatment of malignant gliomas. Neurosurgery 1996; 39: 478-483.

24. Fakhrai, H., Dorigo, O., Shawler, D.L. et al: Eradication of established intracranial rat gliomas by transforming growth factor beta antisense gene therapy. Proc Natl Acad Sci U S A. 1996; 93: 2909-2914.

25. Farag, S.S., Fehniger, T.A., Becknell, B., Blaser, B.W., Caligiuri, M.A.: New directions in natural killer cell-based immunotherapy of human cancer. Expert Opin Biol Ther. 2003; 3: 237-250.

26. Farkkila, M., Jaaskelainen, J., Kallio, M. et al: Randomised, controlled study of intratumoral recombinant $\gamma$-interferon treatment in newly diagnosed glioblastoma. Br J Cancer 1994; 70: 138-141.

27. Friedberg, J.W., Neuberg, D., Gribben, J.G. et al: Combination immunotherapy with rituximab and interleukin 2 in patients with relapsed or refractory follicular non-Hodgkin's lymphoma. Br J Haematol. 2002; 117: 828-834.

28. Glick, R.P., Lichtor, T., Mogharbel, A., Taylor, C.A., Cohen, E.P.: Intracerebral versus subcutaneous immunization with allogenic fibroblasts genetically engineered to secrete interleukin-2 in the treatment of central nervous system glioma and melanoma. Neurosurgery 1997; 41: 898906.

29. Glick, R.P., Lichtor, T., Panchal, R., Mahendra, A., Cohen, E.P.: Treatment with allogeneic interleukin-2 secreting fibroblasts protects against the development of malignant brain tumors. J Neurooncol. 2003; 64: 139-146.

30. Gorelik, L., Flavell, R.A..: Immune-mediated eradication of tumors through the blockade of transforming growth factor-beta signalling in T cells. Nat Med. 2001; 7: 1118- 
31. Hayes, G.M., Woodroofe, M.N. and Cuzner, M.L.: Microglia are the major cell type expressing MHC class II in human white matter. J. Neurol. Sci. 1987; 80: 25-37.

32. Hayes, R.L., Koslow, M., Hiesiger, E.M. et al: Improved long term survival after intracavitary interleukin-2 and lymphokine-activated killer cells for adults with recurrent malignant glioma. Cancer 1995; 76: 840-852.

33. Held-Feindt, J., Mentlein, R.: CD70/CD27 ligand, a member of the TNF family, is expressed in human brain tumors. Int J Cancer 2002; 98: 352-356.

34. Herrlinger, U., Kramm, C.M., Johnston, K.M. et al: Vaccination for experimental gliomas using GM-CSFtransduced glioma cells. Cancer Gene Ther. 1997; 4: 345352 .

35. Hickey, W.F.: Leukocyte traffic in the Central Nervous System: the participants and their roles. Semin Inmunol. 1999; 11: 125-137.

36. Holladay, F.P., Heitz-Turner, T., Bayer, W.L., Wood, G.W.: Autologous tumor cell vaccination combined with adoptive cellular immunotherapy in patients with grade III/IV astrocytoma. J Neurooncol. 1996; 27: 179-189.

37. Hsu, F.J., Benike, C., Fagnoni, F., Liles, T.M. et al: Vaccination of patients with B-cell lymphoma using autologous antigen-pulsed dendritic cells. Nat Med. 1996; 2: 5258.

38. Huber, D., Philipp, J., Fontana, A.: Protease inhibitors interfere with the transforming growth factor-beta-dependent but not the transforming growth factor-beta-independent pathway of tumor cell-mediated immunosuppression. J Immunol. 1992; 148: 277-284.

39. Inge, T.H., McCoy, K.M., Susskind, B.M., Barrett, S.K., Zhao, G., Bear, H.D.: Immunomodulatory effects of transforming growth factor-beta on lymphocytes. Induction of CD8 expression in the CTLL-2 cell line and in normal thymocytes. J Immunol. 1992; 148: 3847-3856.

40. Ingram, M., Jacques, S., Freshwater, D.B., Techy, G.B., Sheldon, H., Helsper, J.T.: Salvage immunotherapy of malignant glioma. Arch Surg. 1987; 122: 1483-1486.

41. Iwasaki, K., Rogers, L.R., Estes, M.L., Barna, B.P.: Modulation of proliferation and antigen expression of a cloned human glioblastoma by interleukin-4 alone and in combination with tumor necrosis factor-alpha and/or interferon-gamma. Neurosurgery 1993; 33: 489-494.

42. Jeffes, E.W.B., Beamer, Y.B., Jacques, S. et al.: Therapy of recurrent high grade gliomas with surgery, and autologous mitogen activated IL-2 stimulated killer (MAK) lymphocytes. I. Enhancement of MAK lytic activity and cytokine production by PHA and clinical use of PHA. J Neurooncol. 1993; 15: 141-155.

43. Jensen, RL.: Growth factor-mediated angiogenesis in the malignant progression of glial tumors: a review. Surg Neurol. 1998; 49: 189-195.
44. Jereb, B., Petric, J., Lamovec, J., Skrbec, M., Soss, E.: Intratumor application of human leukocyte interferon- $\alpha$ in patients with malignant brain tumors. Am J Clin Oncol. 1989; 12: 1-7.

45. Jereb, B., Petric-Grabnar, G., Klun, B., Lamovec, J., Skrbec, M., Soos, E.: Addition of IFN-alpha to treatment of malignant brain tumors. Acta Oncol. 1994; 33: 651-654.

46. Jung, G., Brandl, M., Eisner, W. et al.: Local immunotherapy of glioma patients with a combination of 2 bispecific antibody fragments and resting autologous lymphocytes: evidence for in situ T-cell activation and therapeutic efficacy. Int J Cancer 2001; 91: 225-230.

47. Kehrl, J.H., Roberts, A.B., Wakefield, L.M. et al.: Transforming growth factor beta is an important immunomodulatory protein for human B lymphocytes. J Immunol. 1986; 137: 3855-3860.

48. Kehrl, J.H., Wakefield, L.M., Roberts, A.B. et al.: Production of transforming growth factor beta by human $\mathrm{T}$ lymphocytes and its potential role in the regulation of $\mathrm{T}$ cell growth. J Exp Med. 1986; 163: 1037-1050.

49. Koh, C.Y., Blazar, B.R., George, T. et al: Augmentation of antitumor effects by NK cell inhibitory receptor blockade in vitro and in vivo. Blood 2001; 97: 3132-3137.

50. Kruse, C.A., Cepeda, L., Owens, B., Johnson, S.D., Stears, J., Lillehei, K.O.: Treatment of recurrent glioma with intracavitary alloreactive cytotoxic $\mathrm{T}$ lymphocytes and interleukin-2. Cancer Immunol Immunother. 1997; 45: 77-87.

51. Kruse, C,A, Visonneau, S., Kleinschmidt-DeMasters, B., Gup, C.J., Gómez, G.G., Paul, D.B., Santoli, D.: The human Leucemic T-Cell line, TALL-104, is cytotoxic to human malignant brain tumors and traffics through brain tissue: implications for local adoptive immunotherapy. Cancer Research 2000; 60: 5731-5739.

52. Kuan, C.T., Wikstrand, C.J., Bigner, D.D.: EGFRvIII as a promising target for antibody-based brain tumor therapy. Brain Tumor Pathol. 2000; 17: 71-78.

53. Kuan, C.T., Wikstrand, C.J., Archer, G. et al: Increased binding affinity enhances targeting of glioma xenografts by EGFRvIII-specific scFv. Int J Cancer. 2000; 15; 88: 962969.

54. Kuppner, M.C., Sawamura, Y., Hamou, M.F. et al.: Influence of PGE2- and cAMP-modulating agents on human glioblastoma cell killing by interleukin-2-activated lymphocytes. J Neurosurg. 1990; 72: 619-625.

55. Lapeña, P., Isasi, C., Vaquero, J., Martínez, R., Alvarez-Mon, M.: Modulation by interferon alpha of the decreased natural killer activity in patients with glioblastoma. Acta Neurochir (Wien) 1991; 109: 109-113.

56. Laske, D.W., Youle, R.J., Oldfield, E.H..: Tumor regression with regional distribution of the targeted toxin TFCRM107 in patients with malignant brain tumors". Nat Med. 1997; 3: 1362-1368. 
57. Liau, L.M., Black, K.L., Prins, R.M. et al: Treatment of intracranial gliomas with bone marrow-derived dendritic cells pulsed with tumor antigens. J Neurosurg. 1999; 90(6):111524.

58. Liau, L.M., Jensen, E.R., Kremen, T.J. et al.: Tumor immunity within the Central Nervous System stimulated by recombinant Listeria monocytogenes vaccination. Cancer Research 2002; 62: 2287-2293.

59. Liu, C.Y., Apuzzo, M.: 2001: Things to come. Neurosurgery 2001; 49: 765-778.

60. Liu, Y., O’lillehey, K.: Cell-mediated immunotherapy: a new approach to the treatment of malignant glioma. Cancer Control 2003; 10: 138-147.

61. Mahaley, M.S. Jr, Brooks, W.H., Roszman, T.L. et al.: Immunobiology of primary intracranial tumors, part 1: studies of the cellular and humoral general immune competence of brain-tumor patients. J Neurosurg. 1977; 46: 467476.

62. Martínez, R., Vaquero, J., Ramiro, J., García Salazar, F., De Oya, S.: Intratumoral and Intraventricular Human Lymphoblastoid Alpha Interferon (HLBI) for treatment of Glioblastoma Multiforme. Acta Neurochir (Wien) 1989; 100: 46-49.

63. Matyszak, M. K., Perry, V. H.: A comparison of leukocyte responses to heat-killed bacillus Calmette-Guerin in different CNS compartments. Neuropathol. Appl. Neurobiol. 1996; 22: 44-53.

64. Matyszak, M. K.: Inflammation in the CNS: balance between immunological privilege and immune responses: Prog Neurobiol. 1998; 56: 19-35.

65. Medawar, P.B.: Immunity to homologous grafted skin: the fate of skin homografts transplanted to the brain, to subcutaneous tissue and to the anterior chamber of the eye. Br J Exp Pathol. 1948; 29: 58-69.

66. Merchant, R.E., Grant, A.J., Merchant, L.H., Young, H.F.: Adoptive immunotherapy for recurrent glioblastoma multiforme using lymphokine activated killer cells and recombinant interleukin-2. Cancer 1988; 62: 665-671.

67. Merchant, R.E., Merchant L.H., Cook, S.H.S., McVicar, D.W., Young, H.F.: Intralesional infusion of lymphokine activated killer (LAK) cells and recombinant interleukin-2 (rIL2) for the treatment of patients with malignant brain tumor. Neurosurgery 1988; 23: 725-732.

68. Merchant, R.E., Ellison, E.D., Young, H.F.: Immunotherapy for malignant glioma using human recombinant interleukin-2 and activated autologous lymphocytes. J Neurooncol. 1990; 8: 173-188.

69. Merchant, R.E., McVicar, D.W., Merchant, L.H., Young, H.F.: Treatment of recurrent malignant glioma by repeated intracerebral injections of human recombinant interleukin-2 alone or in combination with systemic interferon- $\alpha$. Results of a phase I clinical trial. J Neurooncol. 1992; 12: 7583.
70. Miyamoto, C.T., Brady, L.W., Rackover, M.A. et al: The use of epidermal growth factor receptor-425 monoclonal antibodies radiolabeled with iodine-125 in the adjuvant treatment of patients with high grade gliomas of the brain. Recent Results. Cancer Research 1996; 141: 183-192.

71. Mocellin, S., Rossi, C.R., Lise, M., Marincola, F.M.: Adjuvant immunotherapy for solid tumors: from promise to clinical application. Cancer Immunol Immunother. 2002; 51 : 583-595.

72. Munz, C., Naumann, U., Grimmel, C., Rammensee, H.G., Weller, M.: TGF-B independent induction of immunogenicity by decorin gene transfer in human malignant glioma cells. Eur J Immunol. 1999; 29: 1032-1040.

73. Nestle, F.O., Alijagic, S., Gilliet, M. et al.: Vaccination of melanoma patients with peptide- or tumor lysate-pulsed dendritic cells. Nat Med. 1998; 4: 328-332.

74. Nitta, T., Sato, K., Yagita, H., Okumura, K., Ishii, S.: Preliminary trial of specific targeting therapy against malignant glioma. Lancet 1990; 335: 368-376.

75. Parkney, I., Chao, C., Petruk, K.: Glioma immunology and immunotherapy. Neurosurgery 2000; 46: 778792.

76. Perrin, G., Schnuriger, V., Quiquerez, A.L. et al.: Astrocytoma infiltrating lymphocytes include major $\mathrm{T}$ cell clonal expansions confined to the CD8 subset. Int Immunol. 1999; 11: 1337-1349.

77. Plautz, G.E., Barnett, G.H., Miller, D.W. et al: Systemic $\mathrm{T}$ cell adoptive immunotherapy of malignant gliomas. $\mathrm{J}$ Neurosurg. 1998; 89: 42-51.

78. Prins, R., Liau, L.: Immunology and Immunotherapy in Neurosurgical disease. Neurosurgery 2003; 53: 114-153.

79. Rand, R.W., Kreitman, R.J., Patronas, N., Varricchio, F., Pastan, I., Puri, R.K.: Intratumoral administration of recombinant circularly permuted interleukin-4-Pseudomonas exotoxin in patients with high-grade glioma. Clin Cancer Research 2000; 6: 2157-2165.

80. Ranges, G.E., Figari, I.S., Espevik, T. et al.: Inhibition of cytotoxic $\mathrm{T}$ cell development by transforming growth factor beta and reversal by recombinant tumor necrosis factor alpha. J Exp Med. 1987; 166: 991-998.

81. Reardon, D.A., Akabani, G., Coleman, R.E. et al.: Phase II trial of murine ${ }^{131} \mathrm{I}$-labeled antitenascin monoclonal antibody 81C6 administered into surgically created resection cavities of patients with newly diagnosed malignant gliomas. J Clin Oncol. 2002; 20: 1389-1397.

82. Resnicoff, M., Sell, C., Rubini, M. et al.: Rat glioblastoma cells expressing an antisense RNA to the insulinlike growth factor-1 (IGF-1) receptor are nontumorigenic and induce regression of wild-type tumors. Cancer Research 1994; 54: 2218-2222.

83. Rhines, L.D., Sampath, P., DiMeco, F. et al: Local immunotherapy with Interleukin-2 delivered from biodegradable polymer microspheres combined with intersti- 
tial chemotherapy: a novel treatment for experimental malignant glioma. Neurosurgery 2003; 52: 872-880.

84. Riva, P., Franceschi, G., Frattarelli, M. et al.: Locoregional radioimmunotherapy of high-grade malignant gliomas using specific monoclonal antibodies labeled with ${ }^{90}$ Y: a phase I study. Clin Cancer Res. 1999; 5: 3275s-3280s.

85. Riva, P., Franceschi, G., Frattarelli, M. et al.: ${ }^{131} \mathrm{I}$ radioconjugated antibodies for the locoregional radioimmunotherapy of high-grade malignant glioma: phase I and II study. Acta Oncol. 1999; 38: 351-359.

86. Rosenburg, S.A., Spiess, P., Lafreniere, R.: A new approach to the adoptive immunotherapy of cancer with tumor-infiltrating lymphocytes. Science 1986; 233: 13181321.

87. Rosenberg, S.A., Lotze, M.T., Muul, L.M. et al.: A progress report on the treatment of 157 patients with advanced cancer using lymphokine-activated killer cells and interleukin2 or high-dose interleukin-2 alone. N Engl J Med. 1987; 316 : 889-897.

88. Roszman, T., Elliot, L., Brooks, W.H.: Modulation of T-cell function by gliomas. Immunol Today 1991; 12: 370374.

89. Ruffini, P.A., Rivoltini, L., Silvani, A. et al.: Factors, including transforming growth factor beta, released in the glioblastoma residual cavity, impair activity of adherent lymphokine-activated killer cells. Cancer Immunol Immunother. 1993; 36: 409-416.

90. Ruggery, L., Capanni, M., Urbani, E. et al.: Effectiveness of donor natural killers cell alloreactivity in mismatched hematopoietic transplants. Science 2002; 295: 2097-3100.

91. Sampath, P., Hanes, J., DiMeco, F., Tyler, B.M., Brat, D., Pardoll, D.M., Brem, H.: Paracrine Immunotherapy with interleukin-2 and local Chemotherapy is synergistic in the treatment of experimental brain tumors. Cancer Research 1999; 59: 2107-2114.

92. Sampson, J.H., Crotty, L.E., Lee, S. et al.: Unarmed, tumor-specific monoclonal antibody effectively treats brain tumors. Proc Natl Acad Sci U S A. 2000; 97: 7503-7508.

93. Scheinberg, L.C., Taylor, J.M.: Immunological aspects of brain tumors. En Krayenbühl H., Maspes PE., Sweet WH. (eds.): Progress in neurological surgery. Chicago, Yearbook Medical Publishers Inc. 1968; vol. 2, pp. 267-291.

94. Sobol, R.E., Fakhrai, H., Shawler, D. et al.: Interleukin-2 gene therapy in a patient with glioblastoma. Gene Ther. 1995; 2 : 164-167.

95. Stander, M., Naumann, U., Dumitrescu, L. et al: Decorin gene transfer-mediated suppression of TGF-beta synthesis abrogates experimental malignant glioma growth in vivo. Gene Ther. 1998; 5: 1187-1194.

96. Tahara, H., Zeh, H.J. III, Storkus, W.J. et al.: Fibroblasts genetically engineered to secrete interleukin 12 can suppress tumor growth and induce antitumor immunity to a
2005; 16 : 345-358

murine melanoma in vivo. Cancer Research 1994; 54: 182189.

97. Tjoa, B.A., Simmons, S.J., Bowes, V.A. et al: Evaluation of phase I/II clinical trials in prostate cancer with dendritic cells and PSMA peptides. Prostate 1998; 36: 39-44.

98. Tjuvajev, J., Gansbacher, B., Desai, R. et al: RG-2 glioma growth attenuation and severe brain edema caused by local production of IL-4 and IFN- $\gamma$. Cancer Research 1995; 55: 1902-1910.

99. Tremond-lukast, G.: Advances in molecular therapies in patients with brain tumors. Cancer Control 2003; 10: 125137.

100. Trouillas, P.: Immunology and immunotherapy of cerebral tumors. Current status. Rev Neurol (Paris) 1973; 128: 23-38.

101. Van Meir, E.G.: Cytokines and tumors of the Central Nervous System. Glia 1995; 15: 264-288.

102. Vaquero, J., Martínez, R., Ramiro, J., García Salazar, F., Barbolla, L., Regidor, C.: Immunotherapy of Glioblastoma with Intratumoral administration of autologous Lymphocytes and human Lymphoblastoid Interferon. A further clinical Study. Acta Neurochir (Wien) 1991; 109: 42-45.

103. Walker, P.R., Saas, P., Dietrich, P.Y.: The role of Fas ligand (CD95L) in immune escape: the tumor cell strikes back. J Immunol. 1997; 158: 4521-4524.

104. Walker, P.R., Calzascia, T., de Tribolet, N., Dietrich, P.Y.: T-cells responses in the brain and their relevance for cerebral malignancies. Brain Res Rev. 2003; 42: 97-112.

105. Weng, W., Levy, R.: Rituximab-induced antibody dependent cellular cytotoxicity (ADCC) in follicular nonHodgkin's lymphoma. Blood 2002; 100: 157a.

106. Yamanaka, R., Abe, T., Yajima, N. et al.: Vaccination of recurrent glioma patients with tumour lysate-pulsed dendritic cells elicits immune responses: results of a clinical phase I/II trial. Br J Cancer 2003; 89: 1172-1179.

107. Yamanaka, R., Tsuchiya, N., Yajima, N. et al.: Induction of an antitumor immunological response by an intratumoral injection of dendritic cells pulsed with genetically engineered Semliki Forest virus to produce interleukin-18 combined with the systemic administration of interleukin-12. J Neurosurg. 2003; 99: 746-753.

108. Yamanaka, R., Yajima, N., Abe, T. et al.: Dendritic cell-based glioma immunotherapy (review). Int J Oncol. 2003 Jul; 23: 5-15.

109. Yu, J.S., Wei, M.X., Chiocca, E.A., Mattuza, R.L., Tepper, R.I.: Treatment of glioma by engineered IL-4 secreting cells. Cancer Res. 1993; 53: 3125-3128.

110. Yu, J.S., Wheeler, C.J., Zeltzer, P.M. et al.: Vaccination of malignant glioma patients with peptide-pulsed dendritic cells elicits systemic cytotoxicity and intracranial T-cell infiltration. Cancer Res. 2001; 61: 842-847.

111. Yung, W.K.A., Steck, P.A., Kelleher, P.J. et al.: Growth inhibitory effect of recombinant $\alpha$ and $\beta$ interferon on 
human glioma cells. J Neurooncol. 1987; 5: 320-330.

112. Yung, W.K.A., Castellanos, A.M., Van Tassel, P., Moser, R.P., Marcus, S.G.: A pilot study of recombinant interferon beta (IFN- $\beta$ ser) in patients with recurrent glioma. J Neurooncol. 1990; 9: 29-34.

113. Zeltzer, P.M., Moilanen, B., Yu, J.S., Black, K.L.: Immunotherapy of malignant brain tumors in children and adults: from theoretical principles to clinical application. Child's Nerv Sys. 1999; 15: 514-528.

114. Zhang, E.T., Richards, H.K., Kida, S., Weller, R.O.: Directional and compartimentalised drainage of interstitial fluid and cerebrospinal fluid from the rat brain. Acta Neuropathol. $1992 ; 83: 233-239$.
115. Zuber, P., Kuppner, M.C., de Tribolet, N.: Transforming growth factor-beta 2 down-regulates HLA-DR antigen expression on human malignant glioma cells. Eur J Immunol. 1988; 18: 1623-1626.

Pinilla-Arias, D.; Mateo Sierra, O.; Gutiérrez, F.A.; Fernández-Carballal, C.; Carrillo, R.: Inmunoterapia en astrocitomas de alto grado: principios y estado actual. Neurocirugía 2005; 16: 345-358.

Correspondencia postal: D. Pinilla Arias. Servicio de Neurocirugía. Hospital General Universitario Gregorio Marañón. C /Dr. Esquerdo 46. 28007 Madrid. 